\title{
Addendum to: \\ Pseudo generators for under-resolved molecular dynamics ${ }^{\star}$
}

\section{Eur. Phys. J. Special Topics 224, 2463-2490 (2015) \\ DOI: 10.1140/epjst/e2015-02422-y}
A. Bittracher ${ }^{1, a}$, C. Hartmann ${ }^{2, b}$, O. Junge ${ }^{1, c}$, and P. Koltai ${ }^{2, d}$
${ }^{1}$ Fakultät für Mathematik, Technische Universität München, 85748 Garching, Germany
${ }^{2}$ Institut für Mathematik, Freie Universität Berlin, 14195 Berlin, Germany

We would like to add the following acknowledgement to our article published in Eur. Phys. J. Special Topics, September 2015, Volume 224, Issue 12, pp. 2463-2490.

The research was partially funded by the Deutsche Forschungsgemeinschaft through the Research Center Matheon and the CRC 1114 "Scaling Cascades in Complex Systems" (Projects A05 and B05).

\footnotetext{
* The online version of the orginial article can be found at https://doi.org/10.1140/epjst/e2015-02422-y

a e-mail: bittrach@ma.tum.de

b e-mail: chartman@mi.fu-berlin.de

c e-mail: oj@tum.de

d e-mail: peter.koltai@fu-berlin.de
} 